\title{
Contrast-enhanced endoscopic ultrasound diagnosis of the intraductal papillary mucinous neoplasm
}

\author{
Giovanna Del Vecchio Blanco ${ }^{1}$ (D) Cristina Gesuale ${ }^{1}$. Alessandro Anselmo ${ }^{2}$. Giampiero Palmieri ${ }^{2}$. \\ Francesca Baciorri ${ }^{3} \cdot$ Monia Di Prete $^{3} \cdot$ Giuseppe Tisone $^{2} \cdot$ Giovanni Monteleone $^{1} \cdot$ Omero Alessandro Paoluzi $^{1}$
}

Received: 6 April 2019 / Accepted: 17 June 2019

(c) Japanese Society of Gastroenterology 2019

\begin{abstract}
Pancreatic cystic neoplasms (PCNs) are a frequent incidental finding during an ultrasound or other radiological investigations. As PCNs may have a potential of malignancy, a precise differential diagnosis between a malignant and benign lesion is crucial to define appropriate management of patients with this kind of lesions. Radiology, with computed tomography (CT) and magnetic resonance imaging, may not be conclusive in the diagnostic assessment of PCNs. Endoscopic ultrasound (EUS), a simple and relatively low invasive technique, is able to identify intra-cystic worrisome features suggesting malignancy. Fine-needle aspiration (FNA) of the cystic fluid or of intra-cystic tissue nodule during EUS is an adjunctive procedure for reaching a conclusive diagnosis. As EUS-FNA is burdened by complications, the use of intravenous contrast may increase the diagnostic accuracy of EUS allowing in many cases a correct diagnosis of PCN at high risk of malignancy, without additional risk of complication during the procedure. The present report deals with the case of a cystic lesion found by CT scan in the pancreatic head of a 59-year-old woman suffering from mild epigastric pain. Once submitted to EUS, malignant nature of PCN was suspected due to the finding of a typical worrisome feature, the presence of a mural nodule. The intravenous administration of contrast medium during the EUS confirmed malignancy and the patient was immediately sent to the surgeon for pancreatic resection. Histology revealed an intraductal papillary mucinous neoplasm, with areas of high-grade dysplasia in the main and secondary ducts, progressed toward an invasive carcinoma.
\end{abstract}

Keywords Contrast enhancement · Endoscopic ultrasonography $\cdot$ Intraductal papillary mucinous neoplasm $\cdot$ Pancreatic cystic neoplasm

\section{Introduction}

A pancreatic cystic neoplasm ( $\mathrm{PCN})$ is a frequent incidental finding in the daily activity during an ultrasound or other radiological investigations, such as CT scan or MRI [1]. In most cases, $\sim 90 \%$, PCNs are serous cystadenomas, mucinous cystic neoplasm (MCN) or intraductal papillary mucinous neoplasm (IPMN) [2]. These 'incidentalomas', indeed, represent a relevant clinical challenge as they may have a

Giovanna Del Vecchio Blanco

giovanna.del.vecchio.blanco@uniroma2.it

1 Gastroenterology Unit, Department of Systems Medicine, University of Rome Tor Vergata, 00133 Rome, Italy

2 Transplant Surgery Unit, Department of Surgery, University of Rome Tor Vergata, Rome, Italy

3 Anatomic Pathology Unit, Department of Biomedicine and Prevention, University of Rome Tor Vergata, Rome, Italy potential of malignancy. While serous cystadenoma has no risk of malignancy and generally does not require surgery, the other two types of PCNs have a high risk to progress toward malignancy and warrant surgery. Therefore, an accurate characterization of a PCN is mandatory for discriminating which patients are in need of a rapid surgery in respect to those who can be followed up.

The present report deals with the case of a middle-aged woman with a cystic lesion in the pancreatic head incidentally found by a CT scan and finally revealed to be an invasive carcinoma originating from an intraductal papillary mucinous neoplasm (IPMN), with areas of high-grade dysplasia in the main and secondary ducts. 


\section{Case report}

A 59-year-old woman referred to our gastroenterology unit following the finding of a PCN by a CT scan performed for the presence of abdominal pain and weight loss. At a clinical visit, the patient was not jaundiced, when interviewed she described the abdominal pain as mild, in the epigastric area, persisting from 3 months in absence of nausea, or digestive changes. No history of acute pancreatitis or other relevant diseases was present. Family history was negative for benign or malignant pancreatic disorders. The haematochemical evaluation revealed no change of parameters. The abdominal CT scan, performed with no dedicated pancreatic protocol, revealed a $5 \mathrm{~cm}$ multilocular cystic lesion in the pancreatic head (Fig. 1a), with peripheral contrast enhancement along the cystic wall and no cleavage planes with the duodenal wall and portal vein. The main pancreatic duct (MPD) was normal in the body and tail, with no clear communication with the cystic lesion. Pancreatic parenchyma of body and tail was normal (Fig. 1b). Based on radiological features, a malignant lesion was suspected, compatible with a mucinous pancreatic cystic neoplasm, thus the patient was proposed to undergo endoscopic ultrasound (EUS) with fine-needle aspiration (FNA). After taken written informed consent, the patient underwent EUS (GF-UCT180, Olympus Italia S.r.l., Milan, Italy) on anesthesia with propofol by an anesthesiologist. According to the European guidelines [3] on pancreatic cystic neoplasms, the B-mode EUS described a large $(>40 \mathrm{~mm})$ multiloculated cystic lesion with a maximum diameter $>50 \mathrm{~mm}$ (Fig. 2a) in the pancreatic head. The MPD was not easy to assess due to the compression from the cyst. The remaining pancreatic parenchyma in the body and tail showed normal echostructure with no dilatation of MPD. A mural nodule of $\geq 5 \mathrm{~mm}$ in size was detected (Fig. 2b). A CH-EUS was performed to
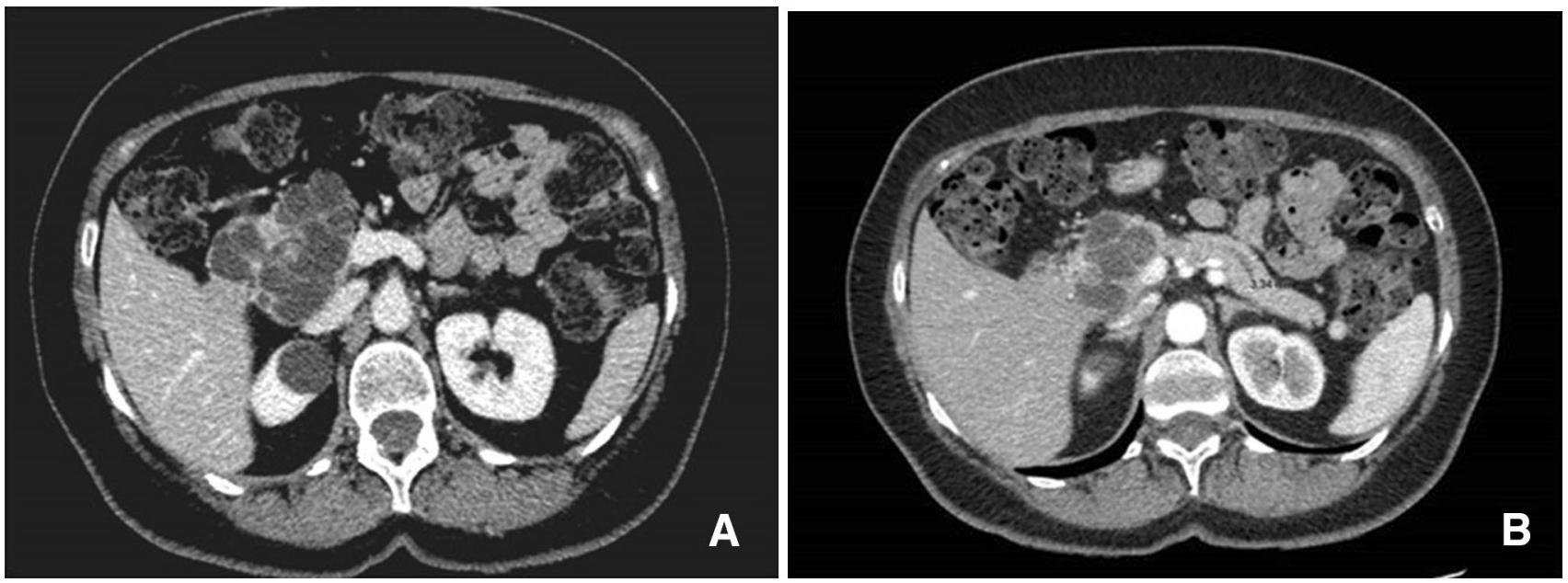

Fig. 1 Computed tomography scan: a A large cystic lesion with peripheral contrast enhancement and wall thickening in the head of the pancreas. b Image of normal pancreatic body and tail, with not dilated main pancreatic duct

Fig. 2 B-mode endoscopic ultrasound image: a, b A multiloculated cystic lesion of $5 \mathrm{~cm}$ with septa and mural nodule. b The white arrow indicates the mural nodule
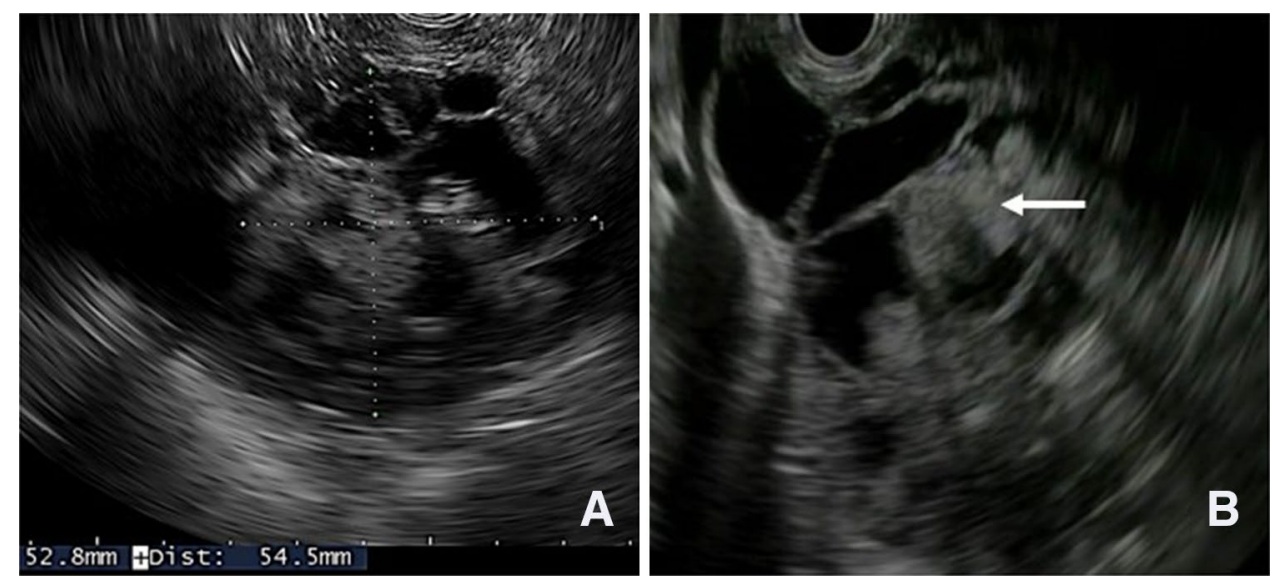
distinguish mural nodule from mucus clot. A sulphur hexafluoride microbubbles (SonoVue ${ }^{\circledR}$, Bracco S.r.l., Milan, Italy) dissolved in $5 \mathrm{~mL}$ of saline solution, was intravenously injected through a $20 \mathrm{G}$ cannula within $2-3 \mathrm{~s}$, followed by a $10 \mathrm{~mL}$ of saline flush. After injecting the contrast medium, a clear hyperenhancement of mural nodule and septations was detected (Fig. 3a, b). The nodule appeared as a large and irregularly surfaced, protruding lesion in the cyst (Fig. 3) and was defined as a type III according to the classification of Ohno et al. [4]. Therefore, a frank malignant transformation of the cystic lesion was evident, and EUS-FNA was considered unnecessary. Following a multidisciplinary team decision, the patient underwent pancreatic resection (Whipple procedure) with regional lymph nodes, gallbladder, and omentum dissection (Fig. 4). Histopathology revealed the presence of foci of a moderately differentiated (G2) invasive adenocarcinoma in a pancreatobiliary IPMN. Moreover, high-grade dysplasia/carcinoma in situ was present both in primary and secondary pancreatic ducts (Figs. 5, 6). Resection margins were not involved by the malignant tumor. All detected lymph nodes, gallbladder and omentum were negative for metastasis. The final pathological staging was pT3N0 based on the UICC-TNM classification (8th edition, 2017).

The postoperative course was uneventful. Three months after the operation, the patient remained in a stable condition with no symptoms of recurrence.

\section{Discussion}

PCNs are incidentally detected with a prevalence of $~ 2 \%$ by CT scan and up to $13-45 \%$ by MRI [1, 3, 5]. According to the classification by the European Study Group on Cystic Tumours of the Pancreas [3], PCNs may be distinguished in epithelial and non-epithelial cysts, which, in turn, may be subclassified in neoplastic and non-neoplastic lesions (Table 1). Excluding the simple cystic and pseudo-cystic forms, most of these pancreatic lesions are

Fig. 3 a B-mode image of the cystic lesion, the white arrow indicatese a mural nodule. b Contrast-enhanced image. The white arrow indicates the enhanced mural nodule of the cyst clearly distinguished from a mucouss clot
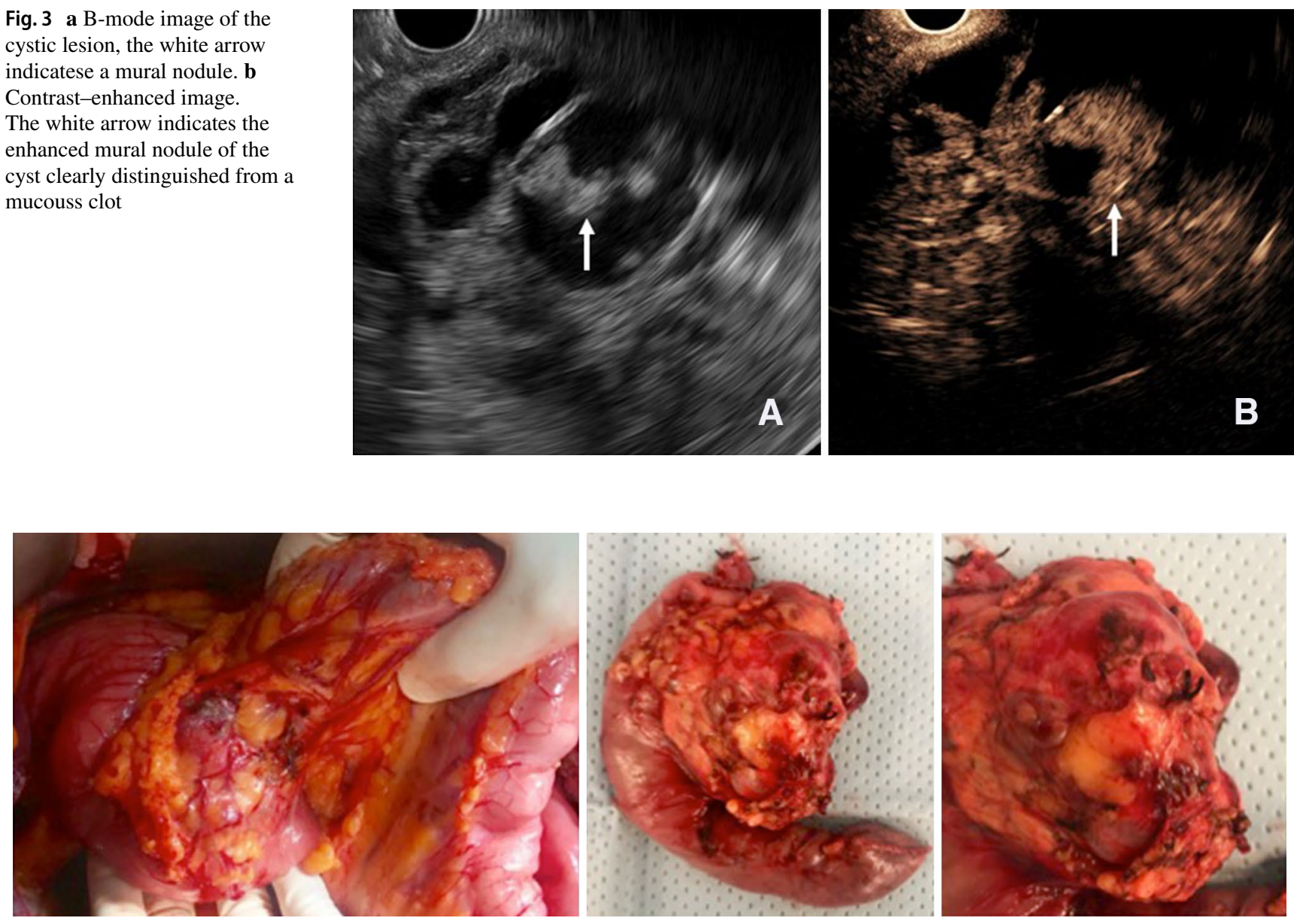

Fig. 4 The surgical specimen after pancreatic Whipple resection 


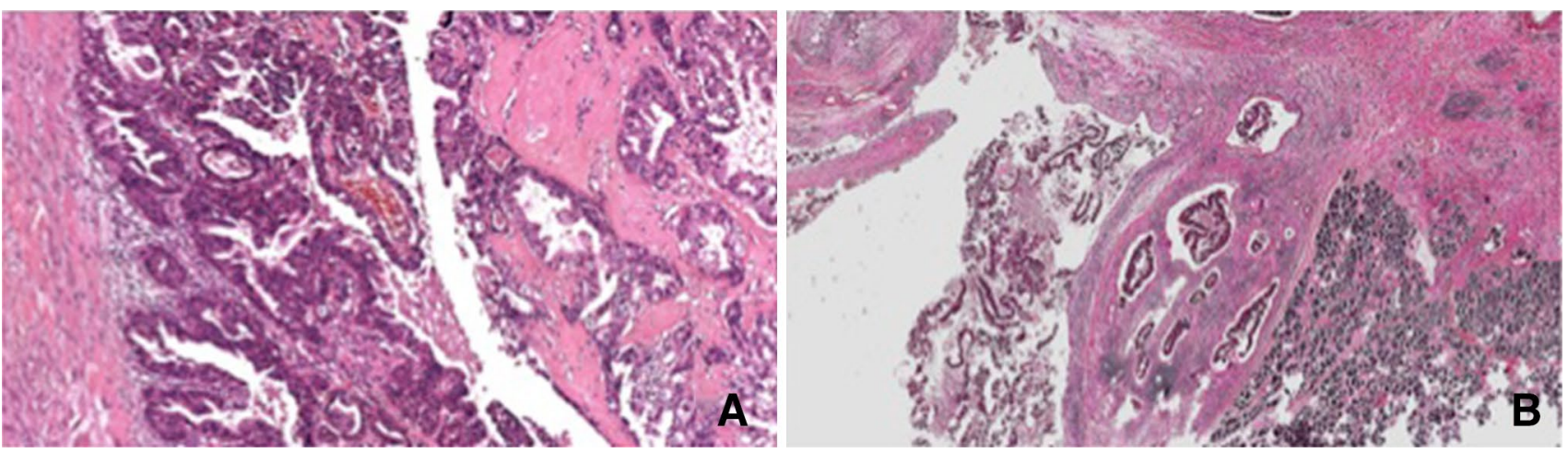

Fig. 5 Histopathology: a an invasive adenocarcinoma with an evident desmoplastic reaction (haematoxylin and eosin staining, original magnification $\times 40$ ). b High-grade dysplasia/carcinoma in situ of the pancreatic ducts (haematoxylin and eosin staining, original magnification $\times 100$ )
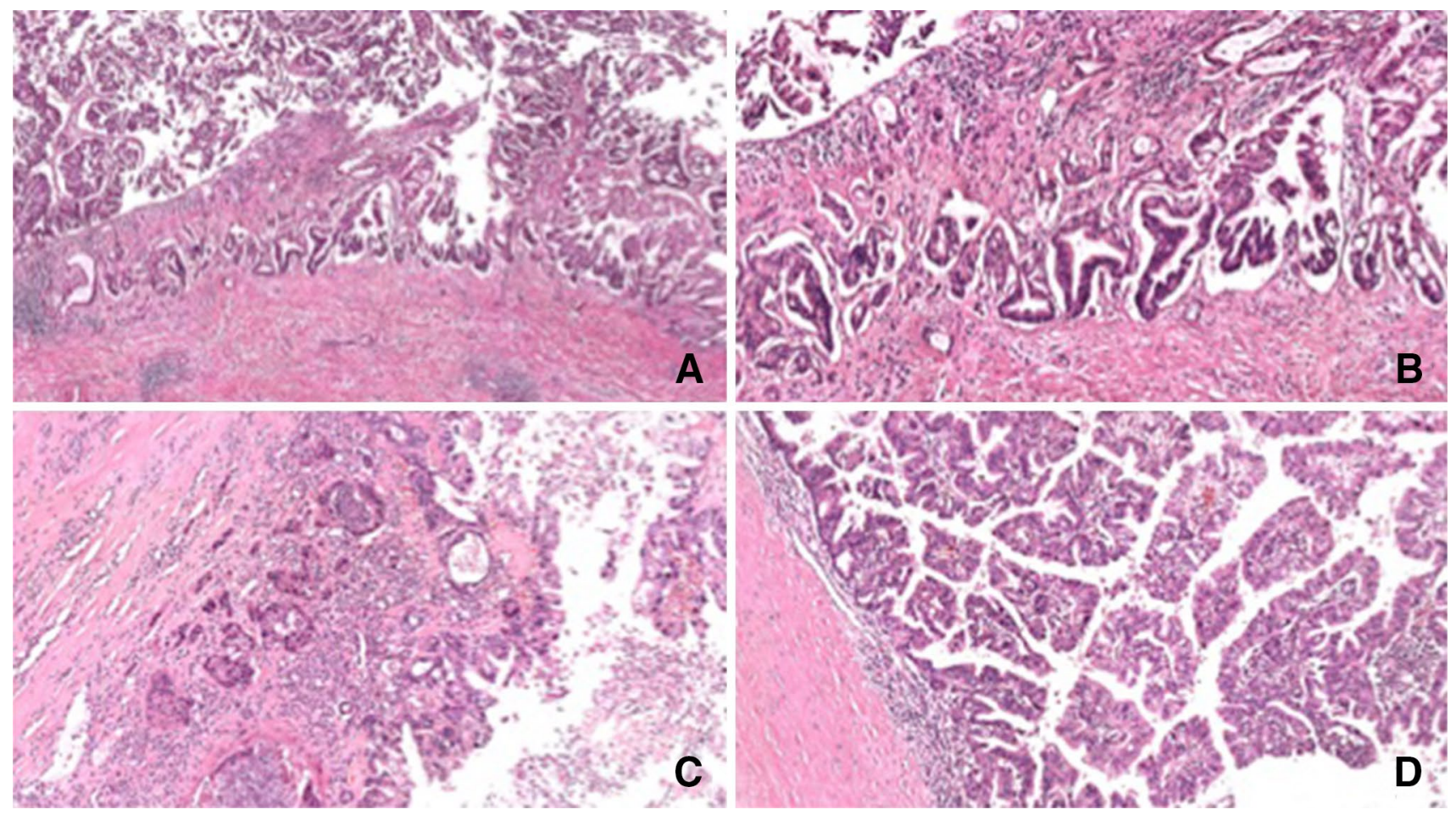

Fig. 6 Histopathology: a-c infiltrative features of an IPMN (haematoxylin and eosin staining, original magnification $\times 100$ a, c, $\times 200 \mathbf{b}$ ). d Papillary aspects of intraductal neoplasia (haematoxylin and eosin staining, original magnification $\times 100$ )

serous cystadenomas, MCN or IPMN, which show a different risk of malignant transformation [3, 5-9] (Table 2). Frequently diagnosed in men between 60 and 70 years of age, IPMNs are located in the pancreatic head in $~ 70 \%$ of the cases. The risk to develop malignancy in case of IPMN ranges from 1.4 to $80 \%$ in the literature [2, 3, 6-9]. Based on histological features and mucin immunophenotype, IPMNs are classified into gastric, intestinal, pancreatobiliary and oncocytic types $[2,10,11]$. The prognosis for pancreatobiliary IPMN is the same as the pancreatic adenocarcinoma which is associated with a high risk of malignancy and post-resection recurrence $[10,11]$. An accurate assessment of IPMN and early detection of worrisome features is crucial in establishing the prognosis and appropriate management of patients with these kinds of lesions. The radiological evaluation by CT scan and MRI is used as the first-line diagnostic approach as these techniques are non-invasive and have a high resolution [3, 5]. However, CT scan and MRI show a diagnostic accuracy varying from 40 to $90 \%$ in identifying the specific 
Table 1 Classification of pancreatic cystic lesions according to European Study Group on cystic tumours of the pancreas. Modified from Ref. [4]

\begin{tabular}{lc}
\hline Epithelial neoplastic & Non-epithelial neoplastic \\
\hline $\begin{array}{l}\text { Mucinous } \\
\text { Intraductal papillary mucinous } \\
\text { neoplasm }\end{array}$ & $\begin{array}{c}\text { Benign non-epithelial neo- } \\
\text { plasm (e.g., lymphangioma) } \\
\text { Mucinous cystic neoplasm }\end{array}$ \\
$\begin{array}{c}\text { Malignant non-epithelial neo- } \\
\text { plasm (e.g., sarcomas) }\end{array}$ \\
$\begin{array}{l}\text { Non Mucinous } \\
\text { Serous cystic neoplasm }\end{array}$ \\
$\begin{array}{l}\text { Serous cystadenocarcinoma } \\
\text { Cystic neuroendocrine tumour }\end{array}$ \\
$\begin{array}{l}\text { G1-2 } \\
\text { Acinar cell cystadenoma }\end{array}$ \\
$\begin{array}{l}\text { Cystic acinar cell carcinoma } \\
\text { Solid pseudopapillary neoplasm }\end{array}$ \\
$\begin{array}{l}\text { Accessory-splenic epidermoid cyst } \\
\text { Cystic hamartoma }\end{array}$ \\
$\begin{array}{l}\text { Cistyc teratoma } \\
\text { Cystic ductal adenocarcinoma }\end{array}$ \\
Cystic pancreatoblastoma \\
Cystic metastatic epithelial neo- \\
plasm
\end{tabular}

Table 2 Risk of malignancy in epithelial mucinous and non-mucinous pancreatic cystic neoplasms according to location and phenotype. Data from Refs. [3-8]

\begin{tabular}{ll}
\hline Type & Risk of malignancy \\
\hline Intraductal papillary mucinous neoplasm & $33-60 \%$ \\
Duct involvement & \\
$\quad$ Side branch & 25.5 \\
Main brunch & $33-60$ \\
Mixed & $33-60$ \\
Phenotype & \\
Gastric & Low \\
Intestinal & Moderate \\
Pancreatobiliary/oncocytic & High \\
Serous cystic neoplasm & - \\
Solid pseudopapillary neoplasm & $10-16 \%$ \\
\hline
\end{tabular}

type of PCN. MRI is indicated as the preferred method in distinguishing between a benign and a malignant PCN [3]. EUS, despite the high number of studies published showing its good diagnostic accuracy in PCNs [12-21], is recommended as an adjunct imaging modality in the European guidelines [3]. Several studies [5, 15-17] have shown that the diameter of the main pancreatic duct or a nodule at the time of diagnosis is a predictor of malignant IPMN. Fine needle aspiration (FNA) is an adjunctive procedure performed during EUS that is helpful in reaching a conclusive diagnosis as it allows the aspiration of cystic fluid or tissue collection of a nodule. The collection of cystic fluid by FNA allows to evaluate the CEA levels, cytology, and KRAS/GNAS mutation analyses, improving the diagnostic accuracy of EUS in differentiating malignant from benign PCN [22]. However, FNA is an invasive technique with a relatively low risk of complications, ranging from 0 to $5 \%[3,5,13,23-25]$, but rarely may lead to death due to fatal acute pancreatitis [24]. Furthermore, the use of FNA may be limited by some contraindications [6]. Intravenous administration of a contrast medium is another ancillary procedure during EUS available in the last decade. After basal B mode EUS scan of the pancreatic gland, the use of contrast medium considerably increases the diagnostic resolution of the EUS in distinguishing malignant from not malignant PCNs on the basis of the enhancement of specific intra-cystic lesions [14-21]. Several studies [15-22] demonstrated that the CE-EUS is able to clearly detect well-known features indicating a malignant transformation, such as the vascularity within the cystic wall, the presence of septations and mural nodule. Furthermore, CE-EUS in PCNs is able to distinguish a mural nodule from mucin plugs, allowing to discriminate when an FNA is indicated. Ohno et al. [4] distinguished four different types of the mural nodule (Table 3) of which type III and type IV were found to be strong predictors of malignancy, with an odds ratio of 10.5. On the basis of this classification, the case presented here was diagnosed to be a PCN with a type III mural nodule. This finding allowed to diagnose a malignancy by CE-EUS, which was confirmed by histopathological examination of the surgical specimen.

Thus, imaging by CE-EUS may allow correct differential diagnosis of the PCNs and in some instances, like the case presented here, may induce to avoid an FNA, reducing the risk of adverse events and costs.

In conclusion, our case confirms the crucial role of the CE-EUS in the assessment of PCNs for revealing the presence of malignant features. As providing crucial findings in the decision-making process in patients with PCNs, the CE-EUS seems to be particularly helpful when FNA is not available or contraindicated. 
Table 3 Classification by Ohno et al. [3] of mural nodule in PCN according to features observed by CE-EUS

\begin{tabular}{ll}
\hline Type & Features \\
\hline Type I (low papillary nodule) & Low, fine, protruding components in the cyst wall or MPD epithelium \\
Type II (polypoid nodule) & A smooth-surfaced component protruding into the cyst or MPD \\
Type III (papillary nodule) & A protruding component with a thickened cyst wall or MPD epithe- \\
& lium or with an irregular, villous structure \\
Type IV (invasive nodule) & A lesion in which papillary nodules were connected to a hypoechoic \\
& area ill-defined from the pancreatic parenchyma \\
\hline
\end{tabular}

\section{Compliance with ethical standards}

Conflict of interest Giovanna Del Vecchio Blanco, Cristina Gesuale, Alessandro Anselmo, Giampiero Palmieri, Francesca Baciorri, Monia Di Prete, Giuseppe Tisone, Giovanni Monteleone, Omero Alessandro Paoluzi declare that they have no conflict of interest.

Human/animal rights All procedures followed have been performed in accordance with the ethical standards laid down in the 1964 Declaration of Helsinki and its later amendments.

Informed consent Informed consent was obtained from all patients for being included in the study.

\section{References}

1. Laffan TA, Horton KM, Klein AP, et al. Prevalence of unsuspected pancreatic cysts on MDCT. Am J Roentgenol. 2008;191:802-7.

2. Castellano-Megías VM, Andrés CI, López-Alonso G, et al. Pathological features and diagnosis of intraductal papillary mucinous neoplasm of the pancreas. World J Gastrointest Oncol. 2014;6:311-24.

3. The European Study Group on Cystic Tumours of the pancreas. European evidence-based guidelines on pancreatic cystic neoplasms. Gut. 2018;67:789-804.

4. Ohno E, Hirooka Y, Itoh A, et al. Intraductal papillary mucinous neoplasms of the pancreas: differentiation of malignant and benign tumors by endoscopic ultrasound findings of mural nodules. Ann Surg. 2009;249:628-34.

5. Italian Association of Hospital Gastroenterologists and Endoscopists, Italian Association for the Study of the Pancreas, Buscarini E, et al. Cystic Pancreatic Neoplasm Study Group. Italian consensus guidelines for the diagnostic work-up and follow-up of cystic pancreatic neoplasms. Dig Liver Dis 2014;46:479-93.

6. Lafemina J, Katabi N, Klimstra D, et al. Malignant progression in IPMN: a cohort analysis of patients initially selected for resection or observation. Ann Surg Oncol. 2013;20:440-7.

7. Kim MJ, Choi DW, Choi SH, et al. Surgical treatment of solid pseudopapillary neoplasms of the pancreas and risk factors for malignancy. Br J Surg. 2014;101:1266-71.

8. Tanaka M, Fernández-del Castillo C, Adsay V, et al. International consensus guidelines 2012 for the management of IPMN and MCN of the pancreas. Pancreatology. 2012;12:183-97.

9. Furukawa T, Klöppel G, Volkan Adsay N, et al. Classification of types of intraductal papillary-mucinous neoplasm of the pancreas: a consensus study. Virchows Arch. 2005;447:794-9.

10. Distler M, Kersting S, Niedergethmann M, et al. Pathohistological subtype predicts survival in patients with intraductal papillary mucinous neoplasm (IPMN) of the pancreas. Ann Surg. 2013;258:324-30.

11. Nakai $Y$, Isayama $H$, Itoi $T$, et al. Role of endoscopic ultrasonography in pancreatic cystic neoplasms: where do we stand and where will we go? Dig Endosc. 2014;26:135-43.

12. Barresi L, Tarantino I, Granata A, et al. Pancreatic cystic lesions: how endoscopic ultrasound morphology and endoscopic ultrasound fine needle aspiration help unlock the diagnostic puzzle. World J Gastrointest Endosc. 2012;4:247-59.

13. Piscaglia F, Nolsøe C, Dietrich CF, et al. The EFSUMB guidelines and recommendations on the clinical practice of contrast enhanced ultrasound (CEUS): update 2011 on non-hepatic applications. Ultraschall Med. 2012;33:33-59.

14. Ohno E, Itoh $\mathrm{A}$, Kawashima $\mathrm{H}$, et al. Malignant transformation of branch duct-type intraductal papillary mucinous neoplasms of the pancreas based on contrast-enhanced endoscopic ultrasonography morphological changes: focus on malignant transformation of intraductal papillary mucinous neoplasm itself. Pancreas. 2012;41:855-62.

15. Yamashita Y, Ueda K, Itonaga M, et al. Usefulness of contrastenhanced endoscopic sonography for discriminating mural nodules from mucous clots in intraductal papillary mucinous neoplasms: a single-center prospective study. J Ultrasound Med. 2013;32:61-8.

16. Hocke M, Cui XW, Domagk D, et al. Pancreatic cystic lesions: the value of contrast-enhanced endoscopic ultrasound to influence the clinical pathway. Endosc Ultrasound. 2014;3:123-30.

17. Harima H, Kaino S, Shinoda S, et al. Differential diagnosis of benign and malignant branch duct intraductal papillary mucinous neoplasm using contrast-enhanced endoscopic ultrasonography. World J Gastroenterol. 2015;21:6252-60.

18. Kamata K, Kitano M, Omoto S, et al. Contrast-enhanced harmonic endoscopic ultrasonography for differential diagnosis of pancreatic cysts. Endoscopy. 2016;48:35-41.

19. Fusaroli P, Serrani M, De Giorgio R, et al. Contrast harmonicendoscopic ultrasound is useful to identify neoplastic features of pancreatic cysts. Pancreas. 2016;45:265-8.

20. Fujita M, Itoi T, Ikeuchi N, et al. Effectiveness of contrastenhanced endoscopic ultrasound for detecting mural nodules in intraductal papillary mucinous neoplasm of the pancreas and for making therapeutic decisions. Endosc Ultrasound. 2016;5:377-83.

21. ASGE Standards of Practice Committee, Early DS, Acosta RD, et al. Adverse events associated with EUS and EUS with FNA. Gastrointest Endosc. 2013;77:839-43.

22. Faias S, Duarte M, Albuquerque C, et al. Clinical impact of KRAS and GNAS analysis added to CEA and cytology in pancreatic cystic fluid obtained by EUS-FNA. Dig Dis Sci. 2018;63:2351-61.

23. Polkowski M, Larghi A, Weynand B, et al. Learning, techniques, and complications of endoscopic ultrasound (EUS)-guided sampling in gastroenterology: European Society of Gastrointestinal Endoscopy (ESGE) Technical Guideline. Endoscopy. 2012;44:190-206. 
24. Vilas-Boas F, Macedo G. Pancreatic cystic lesions: new endoscopic trends in diagnosis. J Clin Gastroenterol. 2018;52:13-9.

25. Jonkman EF, van Tuyl BA, Sanders FB, et al. Severe acute pancreatitis after EUS-FNA of a pancreatic cyst: a rare, but serious complication BMJ Case Rep 2015;2015:bcr2015209442.
Publisher's Note Springer Nature remains neutral with regard to jurisdictional claims in published maps and institutional affiliations. 